\title{
دراسة بعض المتغيرات الفسلجية والجزيئية لعقم النساء في محافظة الأنبار- العراق
}

\section{A Study of Some Physiological and Molecular Variables for Infertility Women in} Anbar Province-Iraq

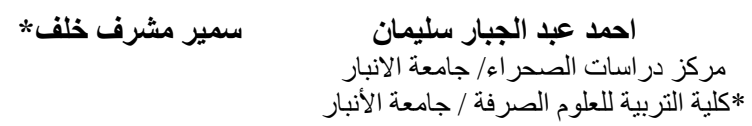

Atekah Q. Qadury

Ahmed A. Suleiman

Samir M. Khalif *

Desart Researh Center/ Anbar University

*College of pure education/ Anbar University

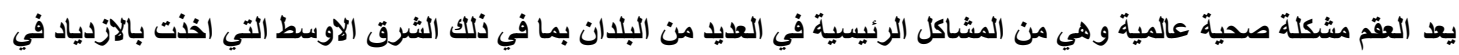

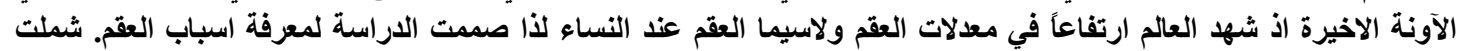

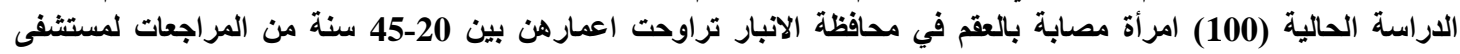

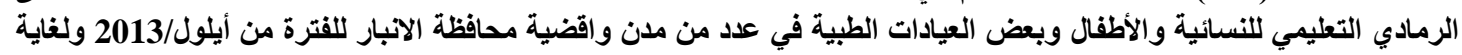

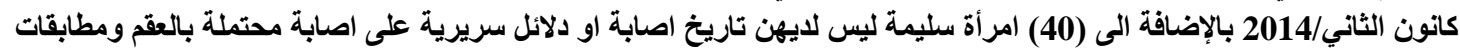

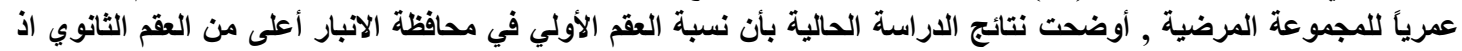

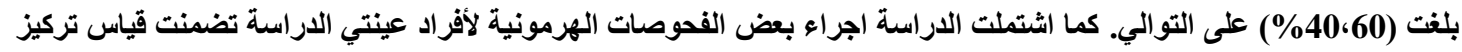

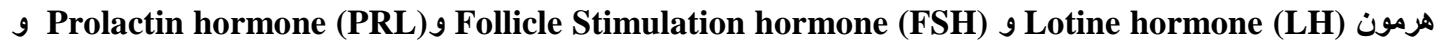
لغرض استبعاد اي عينة تعاني من اضطرابات هرمونية وتبين حدوث ارتفاع معنوي

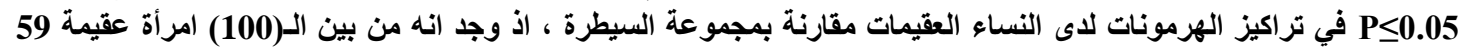

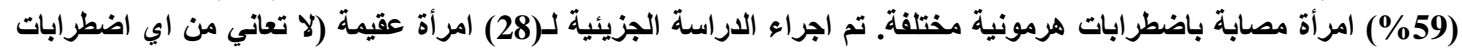

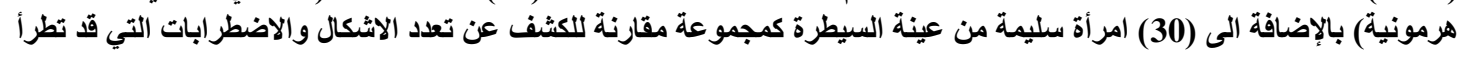

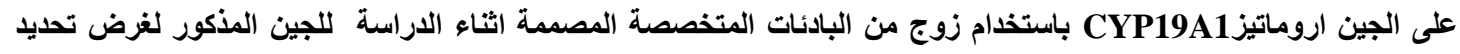

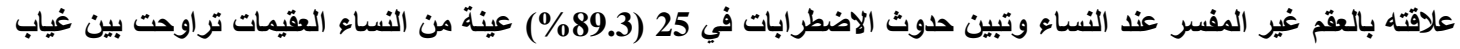

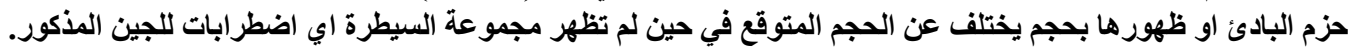

Abstract

الكلمات الدالة: العقم، الهرمون، جين PCR،CYP19A1

Infertility is a global health problem and one of the major problems in many countries, including Middle East, this phenomenon grow in recent years, as the world has seen a rise in the rates of infertility, especially women infertility, so this study conducted to find some reasons of unexplained women infertility. The current study included (100) infertile women in Anbar province, the ages ranged from 20-45 years of revisions to Al-Ramadi Education Hospital for women and pediatric and some medical clinics in a number of cities and districts of Anbar province, through the period from September / 2013 to January / 2014, in addition to (40) healthy women who did not have any history or clinical signs of infertility and their ages matches of the study group, the results of the current study showed that the proportion of primary infertility in Anbar province, the highest of secondary infertility, amounting to $60 \%$ and $40 \%$ respectively. The study also included some hormonal tests to members of study and control samples included measurement of Luteal hormone concentration (LH), Follicle Stimulation hormone (FSH), Prolactin hormone (PRL) and Testosterone Hormone (Testo) to excluding any sample suffer from hormonal disorders the study showed a significant increase $\mathbf{P} \leq \mathbf{0 . 0 5}$ in the concentrations of infertile women hormones as compared with the control group, and it was found among the (100) infertile women that 59 (59\%)women have different hormonal disorders. A molecular study of 28 infertile woman (do not suffer from any hormonal disorders) in addition to (30) healthy women from the control samples for the detection of polymorphism and disorders that may occur to the aromatase gene CYP19A1 using a pair of specific primers designed throughout this study to determining its relationship with unexplained infertility in women, and showing that there disorders in $25(\mathbf{8 9 . 3 \%})$ sample of infertile women ranged from the absence of the primers bands or appearance differs from the expected size while the control group did not show any disturbance.

Keywords: Infertility, hormone, CYP19A1, PCR 


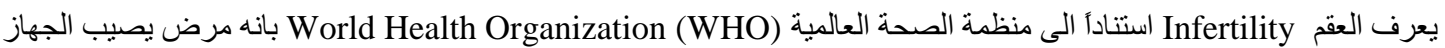

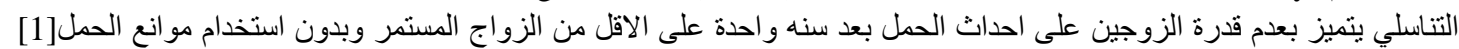

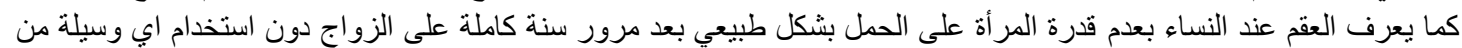

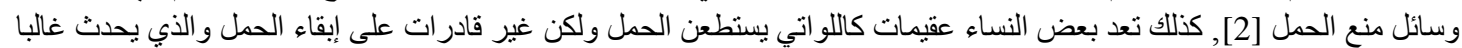

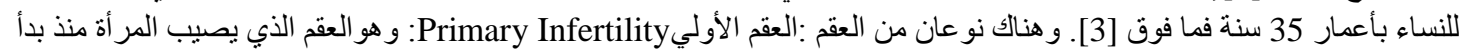

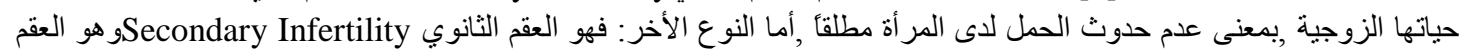

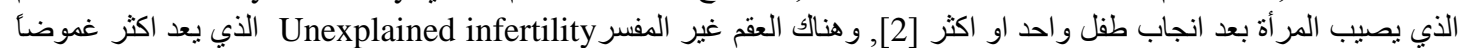

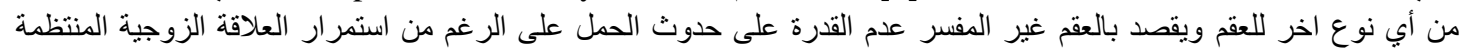

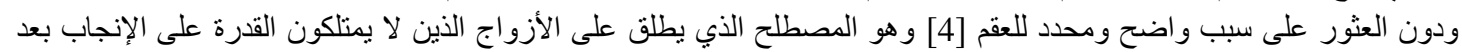

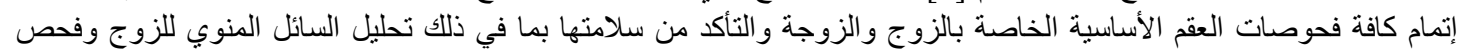

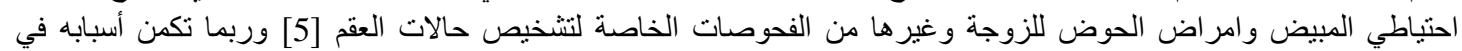

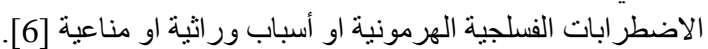

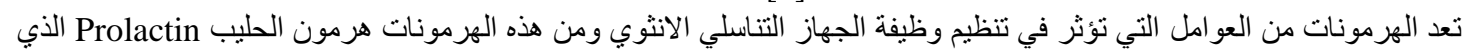

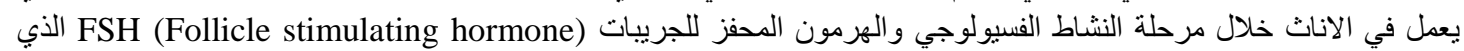

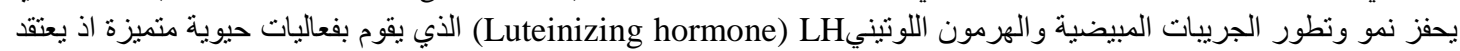

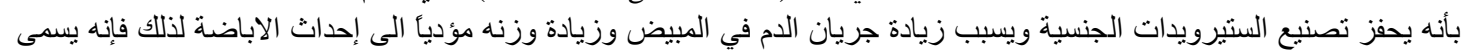

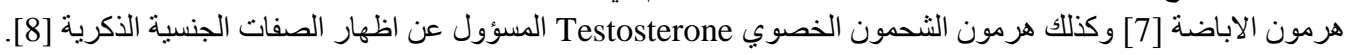

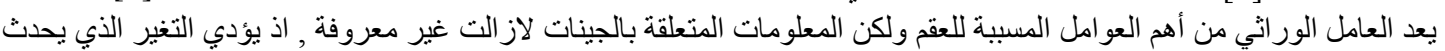

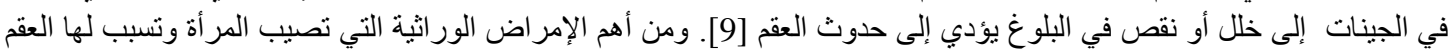

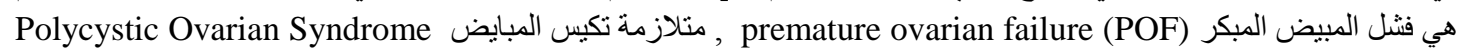
و انتباذ البطانة الرحمية endometriosis (PCOS)

الى حدوث العقم [11]. أما على المستوي الجزيئي فان تعدد اشكال الجينات المشاركة في مسار التخليق الحيوي للهرمونات الستيرويدية ئات

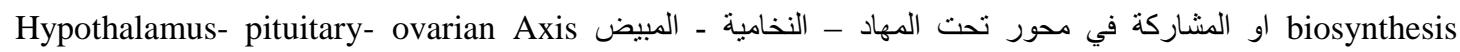

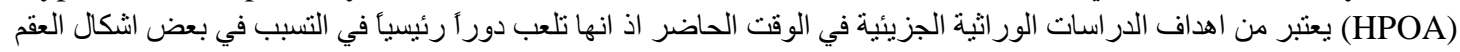

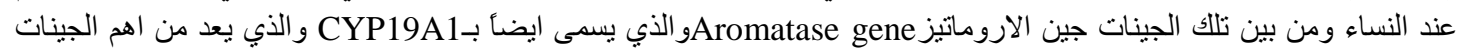

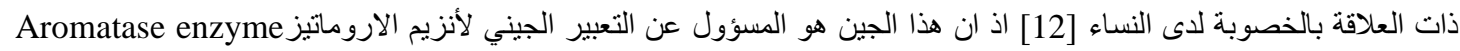

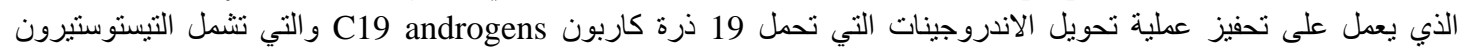
C18 والاندروستينيديون Androstenedione Testosterone

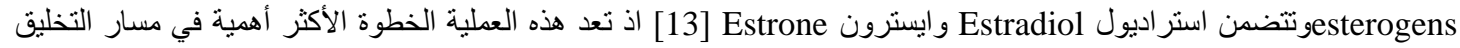

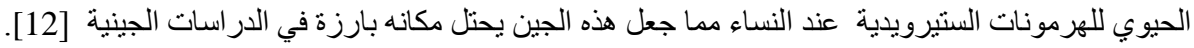

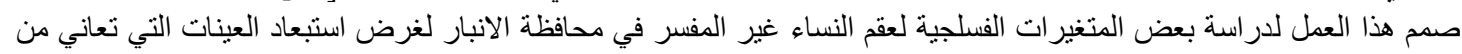

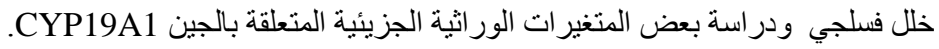

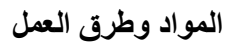

تم جمع عينات الدر اسة من المر اجعات لمستشفى الرمادي التعليمي للنسائية و الأطفال و و العيادات الطبية الخارجية في محافظة الانبار

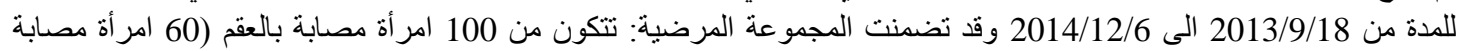

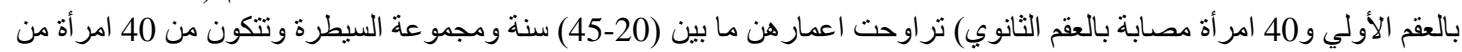

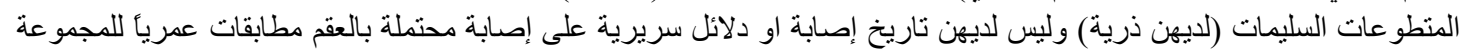

المرضية.

تم قياس تر اكيز الهرمونات (LH, FSH, PRL ,Testo) لجميع عينات الدراسة باتباع الخطوات المرفقة مع عدة التحليل الجاهزة

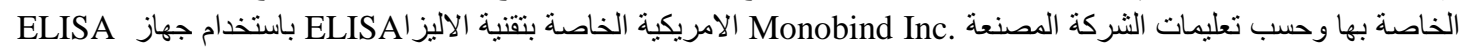

.Reader

تم استخلاص الدنا المجيني من النساء العقيمات الذين لا يعانين من أي اضطر ابات هرمونية اذ كانت مستوى الهرمونات التي تم قياسها

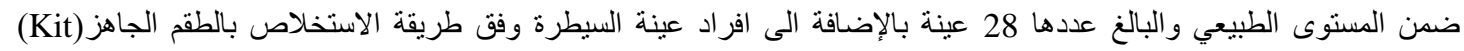

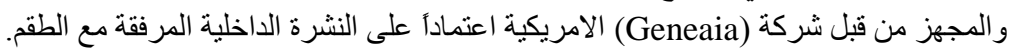

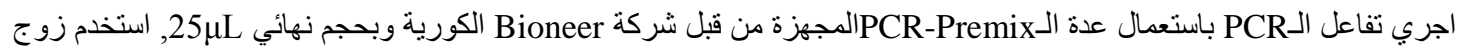

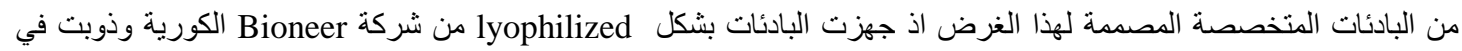
الماء المقطر ونم تحضير نركيز نهائي مقداره 10بيكومول/ميكروليتر . جدول (1) التالي يمثل البادئات المستخدمة في الدراسة. 


\begin{tabular}{|c|c|c|}
\hline 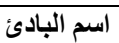 & التتابع Primer sequence & الحجم المتوقعbp \\
\hline SMAP1 & F 5 GCCCTACGAGGGTTCTTCTG & 822 \\
\hline & R 5 GACTGGCACCTGGGGAATAA & \\
\hline SMAP2 & $\begin{array}{l}\text { F } 5 \text { GCCTTTTATTCCCCAGGTGC } \\
\text { R } 5 \text { TCTCTACTGCGCTTGCTCTG }\end{array}$ & 699 \\
\hline
\end{tabular}

تم مزج مكونات التفاعل(PCR-Premix) جيدأ باستخدام جهاز vortex ونبذت لمدة 10 ثواني في جهاز الطرد المركزي

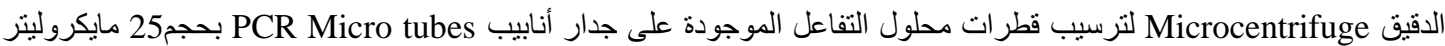

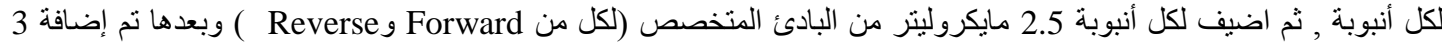

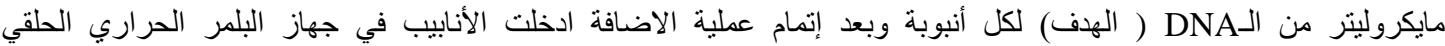

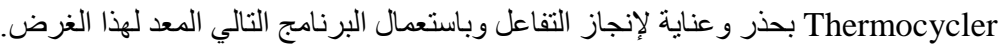

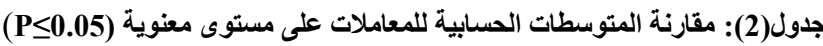

\begin{tabular}{cccc}
\hline Steps & Temperature & Time & Number of cycles \\
Initial denaturation & $94 \mathrm{C}$ & $5 \mathrm{~min}$ & $\mathbf{1}$ \\
Denaturation & $94 \mathrm{C}$ & $1 \mathrm{~min}$ & $\mathbf{3 0}$ \\
Annealing* & $57 \mathrm{C}$ & $1 \mathrm{~min}$ & \\
Extension & $\mathbf{7 2 ~ C}$ & $1 \mathrm{~min}$ & \\
Final extension & $\mathbf{7 2 ~ C}$ & $7 \mathrm{~min}$ & $\mathbf{1}$ \\
\hline
\end{tabular}

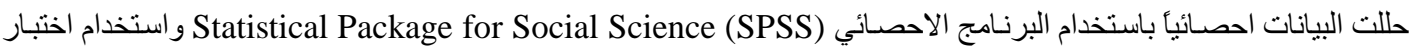

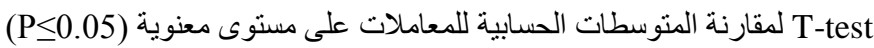
النتائج

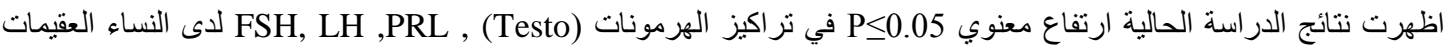

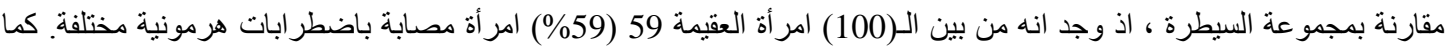

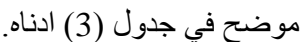

\begin{tabular}{|c|c|c|c|c|c|}
\hline $\begin{array}{c}\text { هرمون Testosterone } \\
\text { ng/ml }\end{array}$ & $\begin{array}{l}\text { هرمون تركيز } \\
\text { Prolactin } \\
\text { ng/ml }\end{array}$ & $\begin{array}{c}\text { تركيز هرمون } \\
\text { LH } \\
\text { mIU/ml }\end{array}$ & $\begin{array}{c}\text { تركيز هرمون } \\
\text { FSH } \\
\text { mIU/ml }\end{array}$ & العراد & المعاملات \\
\hline $1.58 \pm 2.09 * *$ & $19.11 \pm 1.13 *$ & $15.9 \pm 1.66 * *$ & $\begin{array}{c} \pm 1.95 * * \\
13.01\end{array}$ & 60 & العقم الاولي \\
\hline $1.95 \pm 1.11 * *$ & $36 \pm 1.9 * *$ & $14.65 \pm 1.64 * *$ & $\begin{array}{c} \pm 1.33 * * \\
16.47\end{array}$ & 40 & العقم الثانوي \\
\hline $\begin{array}{c}0.45 \pm 0.03 \\
0.2-0.95\end{array}$ & $\begin{array}{c}14.3 \pm 0.78 \\
4.1 \quad \square 28.9\end{array}$ & $\begin{array}{r}\mathbf{6 . 4 9} \pm \mathbf{0 . 4 5} \\
1.7 \quad \square \mathbf{1 3 . 3}\end{array}$ & $\begin{array}{l}6.98 \pm 0.27 \\
4.5 \square 11\end{array}$ & 40 & 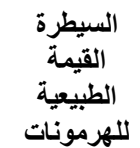 \\
\hline
\end{tabular}

P<0.01 وجود فروق معنوين

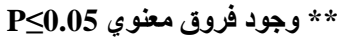

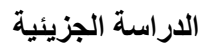

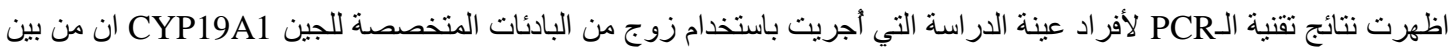

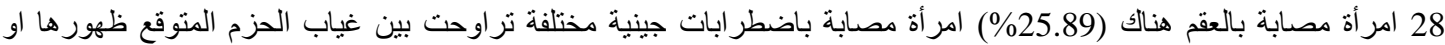

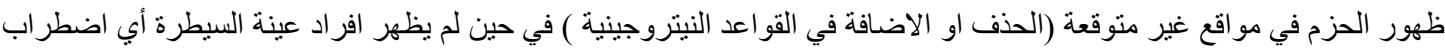

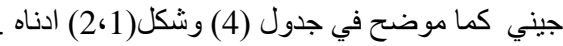
جدول (4): البادئت المستخدمة في الاراسة ,حجم البادئ المتوقع ,وعدد مرات ظهور او غياب الحزمة ونسبتها المنوية لأفراد عينة الاراسة السيطرة.

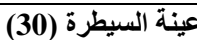
عينة الدراسة (28)

\begin{tabular}{lll} 
bp & & \\
& & \\
822 & SMAP1 & 1 \\
699 & SMAP2 & 2 \\
\hline
\end{tabular}




\section{SMAP1:822}

100

شكل (1) ترحيل الكهربائي لناتج تقنية الـPCR للبادئ رقم

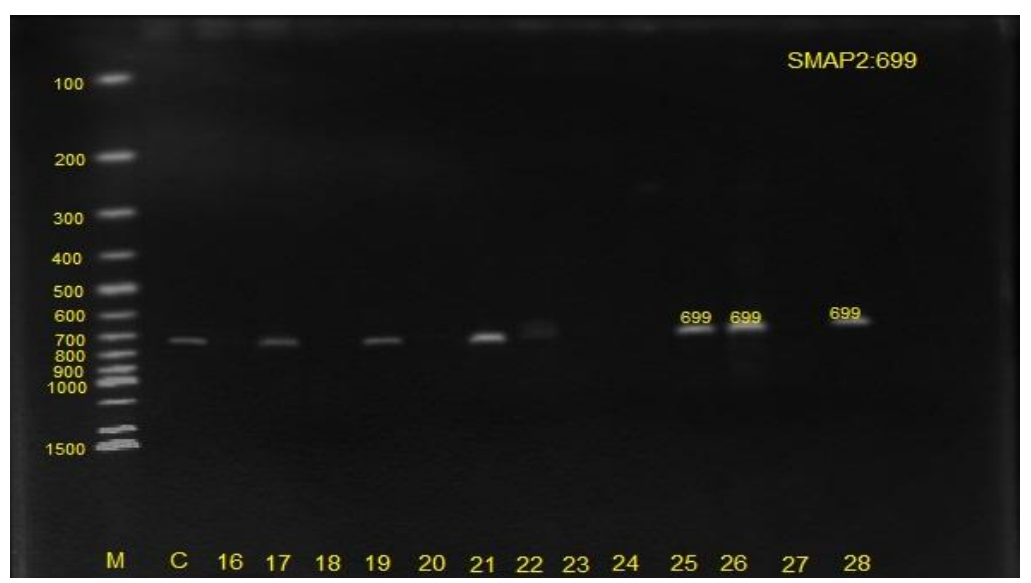

شكل (2) الترحيل الكهربائي لناتج تقتية الـPCR للبادئ رقم SMAP2) لعينات من (16-28)

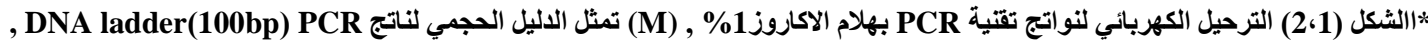
DNA(C)

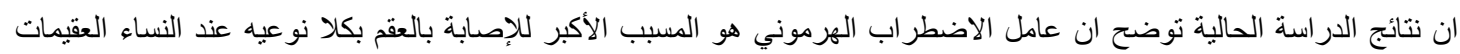

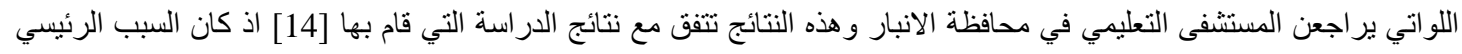

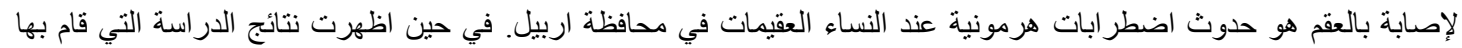

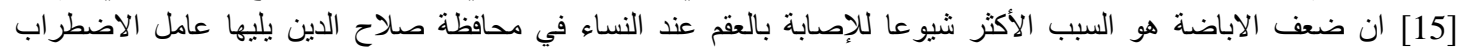

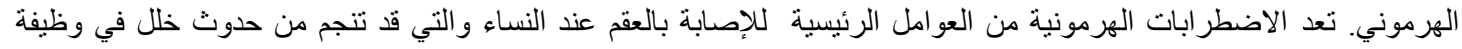

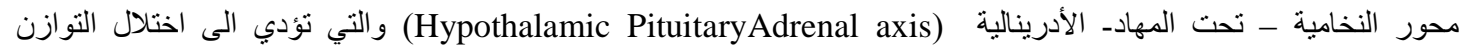

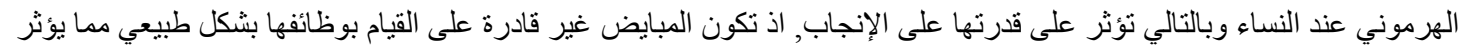

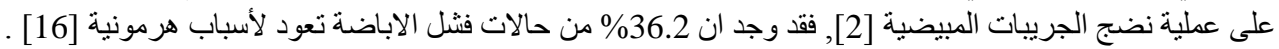

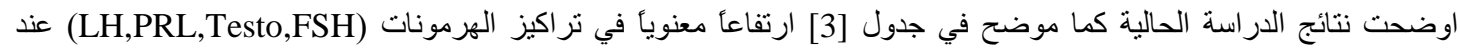

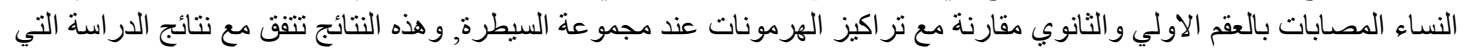

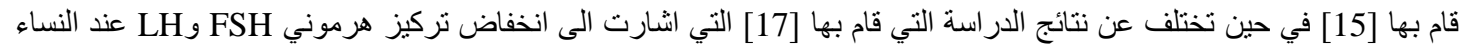

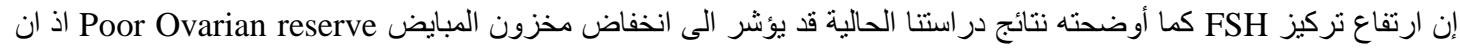

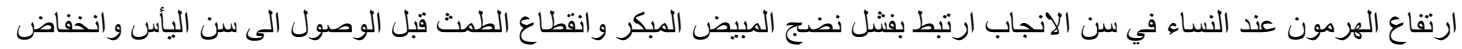

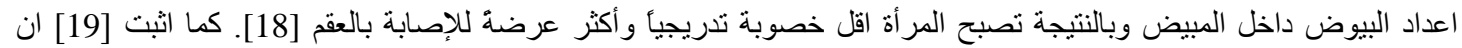

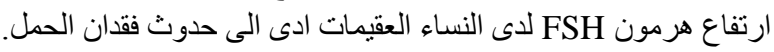

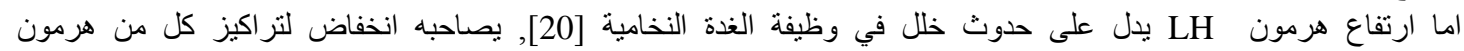

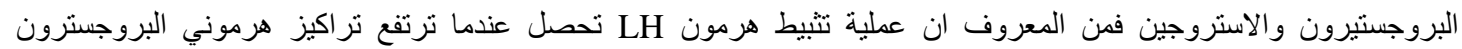

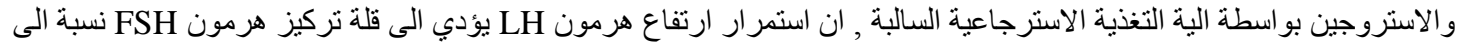

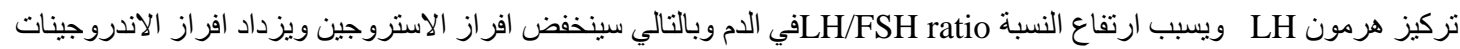
كالهرمون الذكري مما يسبب فثل المبيض في تحفيز نمو ونضج الجريبات المبيضية وعدم تكوين جريبات ناضجة وبنات وبالتالي ندرة

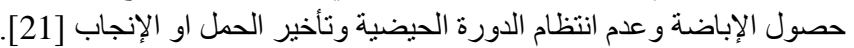


بينت الدر اسة ارتفاع في تركيز هرمون الحليب في الدم للنساء المصابات بالعقم الثانوي تسبب حالة مرضية بطلق عليها بفرط هرمون

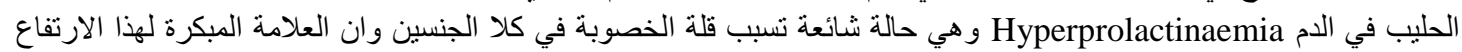

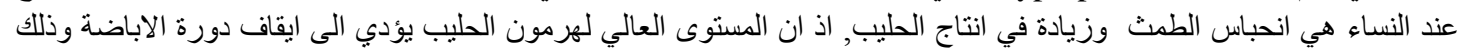

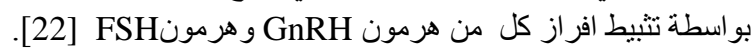

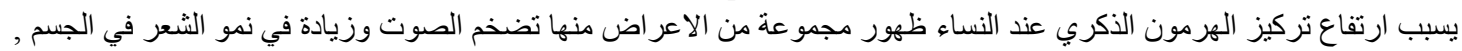

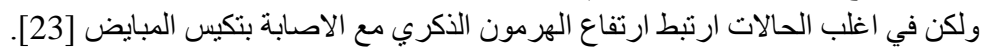

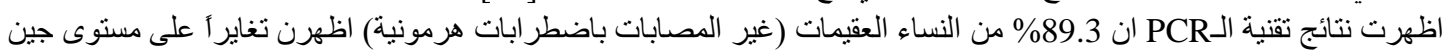

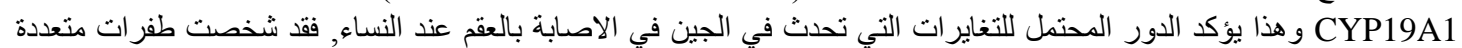

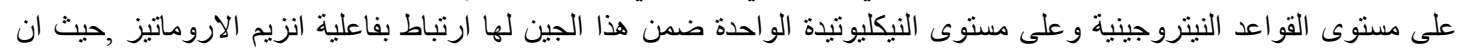

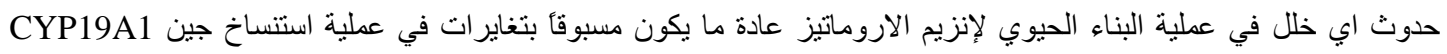

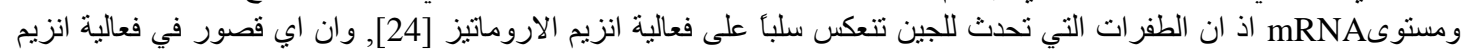

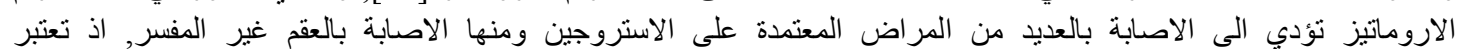

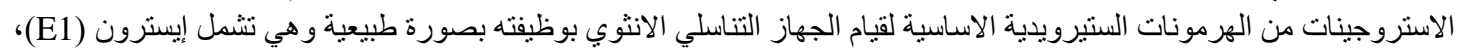

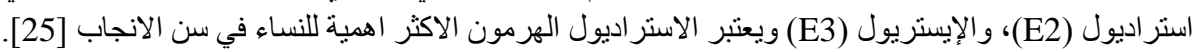

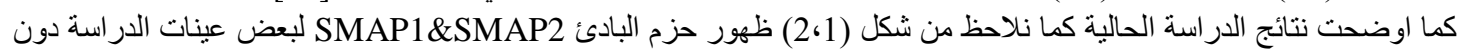

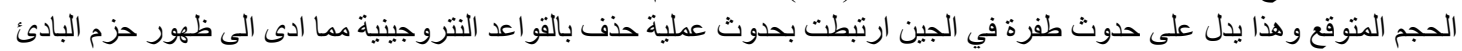

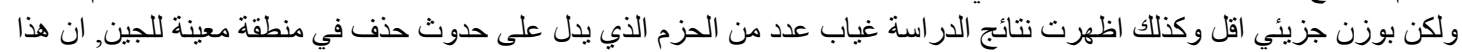

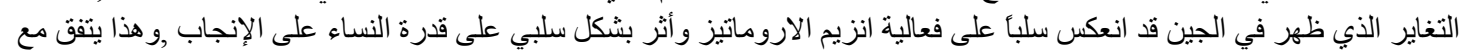

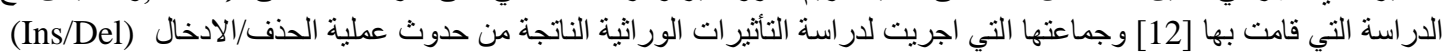
insertion/deletion

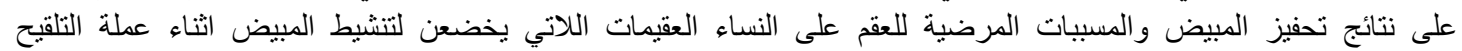

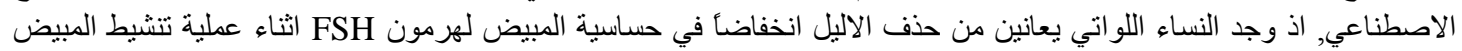
و التي تعكس التغاير الذي حدث في عملية التعبير الجيني والتي تؤثر فعالية انزيم الاروماتيز في في مرحلة مبكرة من عملية تطور

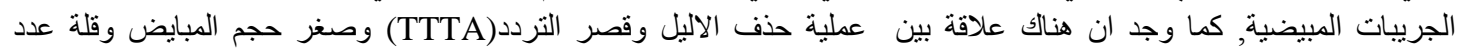

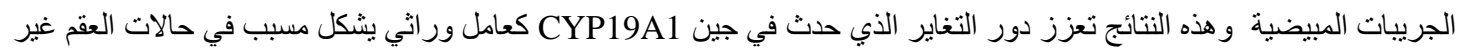

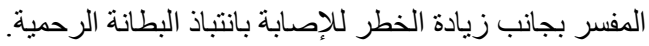

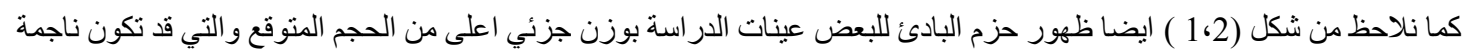

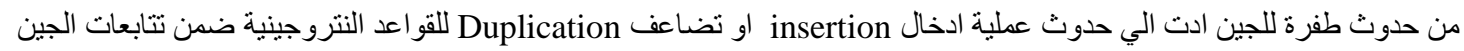

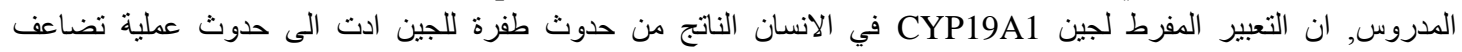

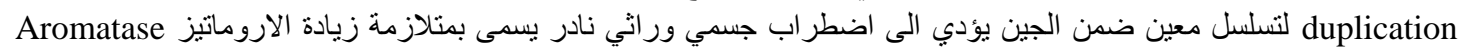
excess syndrome (AEXS)

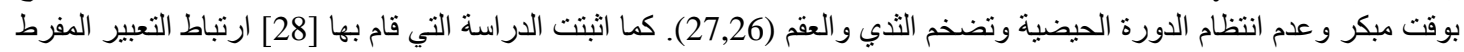

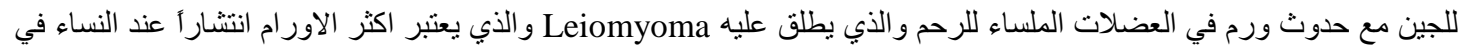

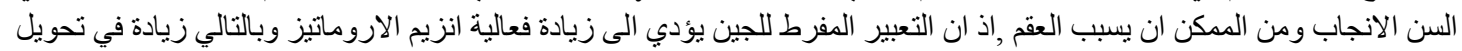

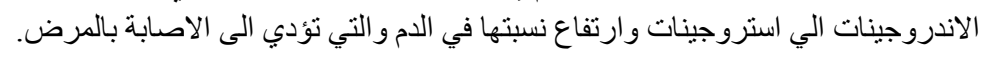

المصادر

1. Mascarenhas, M.N., Flaxman, S.R., Boerma, T., Vanderpoel, S. and Stevens, G.A. (2012). National, regional, and global trends in infertility prevalence since 1990: a systematic analysis of 277 health surveys. PLoS Med. 9(12):e1001356.

2. Olooto, W.E., Amballi, A.A. and Banjo, T.A. (2012). A review of female infertility: important etiological factors and management. J. Microbiol Biotech Res. 2(3):379-385.

3. Sasikumar, S., Shyam, J. S., Madhankumar, E.K., Amburu, P., Kalaiselvi, S., Gopinath, P.M., Dakshayani, D., Krithika, D.J., Chitra, S. and Uma, R. (2014). A study on significant biochemical changes in the serum of infertile women. Int.J.Curr.Res.Aca.Rev. 2 (2):96-115.

4. Rita, I. (2002).Unexplained infertility. Studies on aetiology, treatment options and obstetric outcome. Department of Obstetrics and Gynaecology, Helsinki University Central Hospital.

5. Hatasaka, H. (2011). New perspectives for unexplained infertility. ClinObstet Gynecol. (54):727733.

6. Edward, L. M. (2008). Unexplained Infertility. Fertility Centers of Illinois.

7. Abdennebi, L., Chu, E.Y., Jammes, H., Wei, D. and Remy, J.J. (2003). Maintenance of sexual immaturity in male mice and Bucks by immunization agaist N-Terminal peptides of the folliclestimulating Hormone receptor. Biology of reproduction. 68: 323-327.

8. DiFede, G., Mansueto, P., Pepe, I., Rini, G. B. andCarmina, E. (2010). High prevalence of polycystic ovary syndrome in women with mild hirsutism and no other significant clinical symptoms. Fertility and Sterility.94 (1): 194-197. 
9. Layman, C. (2002). Human gene mutation causing infertility. J.Med.Genet. 39:153-161.

10. Michelle, Z. and Alexander, N.Y. (2013). The Genetics of Infertility: Current Status of the Field. Curr Genet Med Rep. 1:247-260.

11. Matthews, T.J., and Hamilton, B.E. (2009). Delayed childbearing: more women are having their first child later in life. NCHS Data Brief. (21):1-8.

12. Signe, A., Kadri, H., Maire, P., Merli, S., Outi, H., Anneli, S., Agne, V., Helle, K., Andres, M. and Andres, S. (2009). Aromatase gene (CYP19A1) variants, female infertility and ovarian stimulation outcome: apreliminary report. Reproductive BioMedicine Online. (18) 5. 651-657.

13. Sebastian, S. and Bulun, SE. (2001). A highly complex organization of the regulatory region of the human CYP19 (aromatase) gene revealed by the Human Genome Project. J Clin Endocrinol Metab. (86):4600-2.

14. Taha, A.B. and Rashid, K.H. (2013). Etiology of infertility in couples attending maternity hospital in Erbil. Zanco J. Med. Sci.17 (1).

$$
\text { 15. ربد الله, امنه احمد طايس. (2011). در اسة فسلجية, كيموحيوية للدى النساء العقيمات و السليمات في محافظة صلاح الدين. }
$$

16. Beaker, K.L. (2001). Priniciple and practice of endocrinology and Metabolism, $3^{\text {rd }}$ edition, Lippincott Williams and Wikins, Philadelphia. 918-1015.

$$
\begin{aligned}
& \text { 17.عباس، مادلين قاسم. (2008). التأثير الفسلجي لمستويات عدد من الهرمونات ومكونات الدم في النساء العقيمات من المراجعات }
\end{aligned}
$$

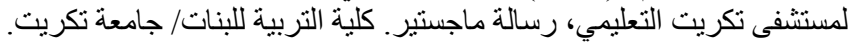

18. Li, Q.z. (2009).TCM Treatment of Female Infertility Caused by High FSH. Journal of the Association of Traditional Chinese Medicine (UK).16:(1).

19. Bhavnani, BR and Stanczyk, FZ. (2012). Misconception and concerns about bioidentical hormones used for custom-compounded hormone therapy. J ClinEndocrinol Metab. 97(3):756-759.

20. Warren, M.P and Stichl, A.l. (1999). Execrcis and female adolscents: effects on the reproductive and skeletal system. J. of American Medical Womens association. 54(3): 115-120.

21. Altuntas, C.Z., Johnson, J.M. and Tuohy, V.K. (2006). Autoimmune targeted disruption of the pituitary-ovarian axis causes premature ovarian failure J. Immunol. 177(3):1988-6.

22. Khalil, H.A., Hanafy, A.M., Saleh ,Y. and Medan, M. (2009).Comparative Changes in Serum Concentrations of Inhibin-B, Gonadotropins and Steroid Hormones at Different Reproductive States in Domestic Turkey Hens. Journal of Reproduction and Development. 55:523-528.

23. Karakurt, F., Sahin, I., Güler, S et al. (2008). Comparison of the clinical efficacy of flutamide and spironolactone plus ethinyloestradiol/cyproterone acetate in the treatment of hirsutism: a randomised controlled study AdvTher. 25 (4): 321-8.

24. Hajar, S., Zivar, S. and Saiedeh, S. (2013). Analysis of aromatase (CYP19) gene in Iranian women with endometriosis. The Egyptian Journal of Medical Human Genetics. (14): 165-169.

25. Gruber, C.J., Tschugguel, W., Schneeberger, C. and Huber, J.C. (2002). Production and Actions of Estrogens. N Engl J Med. (346):340-52.

26. Demura, M., Martin, RM., Shozu, M., Sebastian, S., Takayama, K., Hsu, WT., Schultz, RA., Neely, K., Bryant, M., Mendonca, BB., Hanaki , K., Kanzaki ,S., Rhoads, DB., Misra, M. and Bulun ,SE . (2007). Regional rearrangements in chromosome 15q21 cause formation of cryptic promoters for the CYP19 (aromatase) gene. Hum MolGenet. (16):2529-2541.

27.Binder, G., Iliev, D.I., Dufke, A., Wabitsch, M., Schweizer, R., Ranke, MB. and Schmidt, M. (2005). Dominant transmission of prepubertal gynecomastiadue to serum estrone excess: hormonal, biochemical, and genetic analysis in large kindred. J. ClinEndocrinol Metab. (90):484-492.

28. Shozu, M., Sumitani, H., Segawa, T., Yang, H., Murakami, K., Kasai, T. and Inoue, M. (2002). Over expression of Aromatase P450 in Leiomyoma Tissue Is Driven Primarily through Promoter I.4 of the Aromatase P450 Gene (CYP19). The Journal of Clinical Endocrinology \& Metabolism. 87(6): 25402548 . 